\title{
Coordination Asymmetry of a Dinuclear Copper(II) Complex: Synthesis, Structure, and Magnetism
}

\author{
Takayuki Kayatani, Yoshihito Hayashi, Masatatsu Suzuki, ${ }^{*}$ Katsuhiko Inomata, and Akira Uehara* \\ Department of Chemistry, Faculty of Science, Kanazawa University, Kakuma-machi, Kanazawa 920-11
}

(Received September 5, 1995)

\begin{abstract}
A dicopper(II) complex with a novel unsymmetric dinucleating ligand, $\mathrm{H}_{2} \mathrm{~L}-1$, which can provide donor atom, coordination number, and geometric asymmetries at metal centers, $\left[\mathrm{Cu}_{2}(\mathrm{~L}-1)\left(\mathrm{CH}_{3} \mathrm{COO}\right)\right] \mathrm{ClO}_{4} \cdot \mathrm{H}_{2} \mathrm{O} \cdot 0.5 \mathrm{NaClO}_{4}(\mathbf{1})$, was synthesized, where $\mathrm{H}_{2} \mathrm{~L}-1$ is a racemic 1-bis(2-pyridylmethyl)amino-3-salicylideneaminopropan-2-ol. The ligand is a hybrid one of 1,3-bis[bis(2-pyridylmethyl)amino]propan-2-ol (Htpdp) and 1,3-bis(salicylideneamino)propan-2-ol $\left(\mathrm{H}_{3} \mathrm{~L}\right.$ 2). Crystal structure of 1 was determined by $\mathrm{X}$-ray crystallography. Complex $\mathbf{1}$ crystallizes in monoclinic space group $C$ $2 / c$ with $a=42.704(8), b=12.689(6), c=29.008(9) \AA, \beta=128.28(1)^{\circ}$, and $Z=16$. The crystal structure showed that two stereoisomers (1a and 1b) are present in solid state. This is attributable to the presence of an enantiomeric pair of L-1. Dinuclear cations consist of one square-planar site with a $\mathrm{NO}_{3}$ donor set and one five-coordinate site with a $\mathrm{N}_{3} \mathrm{O}_{2}$ donor set, which are linked by an endogenous alkoxide bridge of L- 1 and an exogenous acetate bridge. A powdered sample of 1 shows weak antiferromagnetic interaction (2.60 B.M./Cu $\mathrm{Cu}_{2}$ at 300 and 1.81 B.M./Cu $/ \mathrm{Cu}_{2}$ at $5 \mathrm{~K}$ ). Cryomagnetic data is analyzed using two exchange coupling constants, $J_{1}=-0.18 \mathrm{~cm}^{-1}$ and $J_{2}=-9.05 \mathrm{~cm}^{-1}$. EHMO calculations revealed that the observed weak antiferromagnetic interaction(s) compared with that found for $\left[\mathrm{Cu}_{2}(\mathrm{~L}-2)\left(\mathrm{CH}_{3} \mathrm{COO}\right)\right](3)\left(J=-85 \mathrm{~cm}^{-1}\right)$ is reasonably interpreted in terms of the energy gap and the nature of HOMO and LUMO. Some other physicochemical properties of the complex are discussed in comparison with those of the parent complexes, $\left[\mathrm{Cu}_{2}(\mathrm{tpdp})\left(\mathrm{CH}_{3} \mathrm{COO}\right)\right]^{2+}(\mathbf{2})$ and $\left[\mathrm{Cu}_{2}(\mathrm{~L}-2)\left(\mathrm{CH}_{3} \mathrm{COO}\right)\right](3)$.
\end{abstract}

A variety of symmetric dinucleating ligands which possess two equivalent coordination sites have been well developed, ${ }^{1)}$ whereas unsymmetric dinucleating ligands which provide stereochemically and/or electronically distinct coordination sites are only limited. ${ }^{2-7)}$ Although some symmetric dinucleating ligands, when coordinated to two metal ions, happen to lead to some different coordination environments for each metal ion, the difference is usually small. For some dinuclear iron $^{8)}$ and dinuclear copper ${ }^{9)}$ metalloproteins, it has been shown or proposed that the coordination environments of two metal ions are inequivalent. Therefore it is interesting to develop unsymmetric dinucleating ligands which can provide donor atom, coordination number, and geometric asymmetries at metal centers and to investigate the physicochemical properties of unsymmetric dinuclear metal complexes in comparison with those of the symmetric dinuclear complexes.

There are many dinuclear metal complexes containing 1,3-diaminopropan-2-olate as a bridging skeleton. ${ }^{10-16)}$ The ligands of this type have been shown to be useful for the dinucleation or polynucleation of metal ions with an endogenous alkoxo bridge. Very recently, an unsymmetric dinucleating ligand similar to the present dinucleating ligand which has also 1,3-diaminopropan-2-olate as a bridging skeleton has been reported. ${ }^{7)}$ As our current studies of asymmetric dinuclear metal complexes, ${ }^{5,17)}$ in this study we synthesized an unsymmetric dinucleating ligand $\left(\mathrm{H}_{2} \mathrm{~L}-1\right.$ as shown in Scheme 1$)$, and its dinuclear copper(II) complex $\left[\mathrm{Cu}_{2}(\mathrm{~L}-\right.$
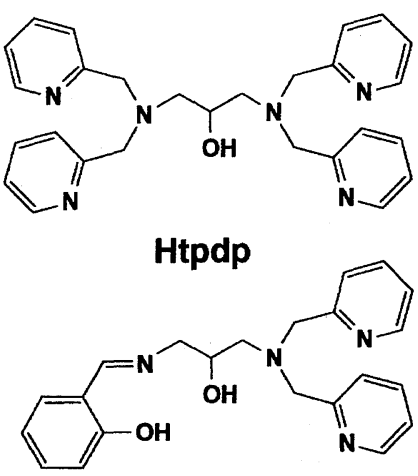

$\mathrm{H}_{2} \mathrm{~L}-1$

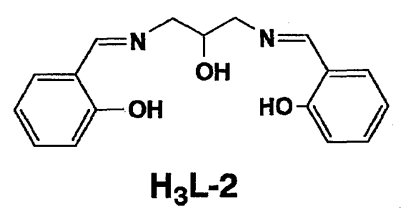

Scheme 1. Ligands.

1) $\left.\left(\mathrm{CH}_{3} \mathrm{COO}\right)\right] \mathrm{ClO}_{4} \cdot \mathrm{H}_{2} \mathrm{O} \cdot 0.5 \mathrm{NaClO}_{4}(\mathbf{1})$. In order to investigate the effect of unsymmetric nature of the present dinucleating ligand, the structural, magnetic, and electrochemical properties of 1 were investigated in comparison with those of the parent complexes with symmetric dinucleating ligands (tpdp and L-2), $\left[\mathrm{Cu}_{2}(\mathrm{tpdp})\left(\mathrm{CH}_{3} \mathrm{COO}\right)\right]\left(\mathrm{ClO}_{4}\right)_{2} \cdot \mathrm{H}_{2} \mathrm{O}(2)$ and $\left[\mathrm{Cu}_{2}(\mathrm{~L}-2)\left(\mathrm{CH}_{3} \mathrm{COO}\right)\right](3) .{ }^{1 \mathrm{lb})}$ 


\section{Experimental}

Preparation of Ligand. $\quad N, N$-Bis(2-pyridylmethyl)-1,3-diaminopropan-2-ol (A). This was prepared in the same way as that described previously. ${ }^{17)}$

Htpdp. This was synthesized according to the literature. ${ }^{15)}$

Preparation of Complexes. $\quad\left[\mathrm{Cu}_{2}(\mathrm{~L}-1)\left(\mathrm{CH}_{3} \mathrm{COO}\right)\right] \mathrm{ClO}_{4}$. $\mathrm{H}_{2} \mathrm{O} \cdot 0.5 \mathrm{NaClO}_{4}$ (1). $\quad \mathrm{N}, \mathrm{N}$-Bis(2-pyridylmethyl)-1,3-diaminopropan-2-ol (A) (0.136 g, $0.5 \mathrm{mmol})$ and salicylaldehyde $(0.061$ $\mathrm{g}, 0.5 \mathrm{mmol})$ were mixed in methanol $\left(3 \mathrm{~cm}^{3}\right)$ with stirring at room temperature. To the resulting solution was added successively copper(II) acetate monohydrate $(0.200 \mathrm{~g}, 1 \mathrm{mmol})$ in methanol (20 $\left.\mathrm{cm}^{3}\right)$, triethylamine $(0.102 \mathrm{~g}, 1 \mathrm{mmol})$, and excess sodium perchlorate $(0.3 \mathrm{~g})$ dissolved in a minimum amount of methanol with stirring. After filtration, the deep green solution was allowed to stand for one day. The deep green crystals deposited were collected by filtration, washed with ethanol and air-dried. Yield: $0.184 \mathrm{~g}$. Anal. Found: C, 38.98; H, 3.68; N, 7.67; Na, 1.52\%. Calcd for $\mathrm{C}_{24} \mathrm{H}_{27} \mathrm{~N}_{4} \mathrm{O}_{11} \mathrm{Cl}_{1.5} \mathrm{Cu}_{2} \mathrm{Na}_{0.5}$ : C, 38.99; $\mathrm{H}, 3.68 ; \mathrm{N}, 7.58 ; \mathrm{Na}, 1.55 \%$.

$\left[\mathrm{Cu}_{2}\right.$ (tpdp) $\left.\left(\mathrm{CH}_{3} \mathrm{COO}\right)\right]\left(\mathrm{ClO}_{4}\right)_{2} \cdot \mathrm{H}_{2} \mathrm{O}$ (2). To a solution of copper(II) acetate monohydrate $(0.200 \mathrm{~g}, 1.0 \mathrm{mmol})$ in methanol $\left(10 \mathrm{~cm}^{3}\right)$ was added a mixture of Htpdp $(0.227 \mathrm{~g}, 0.5 \mathrm{mmol})$ and triethylamine $(0.051 \mathrm{~g}, 0.5 \mathrm{mmol})$ in methanol $\left(10 \mathrm{~cm}^{3}\right)$ with stirring. Excess sodium perchlorate $(0.4 \mathrm{~g})$ dissolved in a minimum amount of methanol was added with stirring. After filtration, the blue solution was allowed to stand for one day. The deep green crystals deposited were collected by filtration, washed with ethanol and airdried. Yield: 0.249 g. Anal. Found: C, 40.95; H, 3.81; N, 10.07\%. Calcd for $\mathrm{C}_{29} \mathrm{H}_{34} \mathrm{~N}_{6} \mathrm{O}_{12} \mathrm{Cl}_{2} \mathrm{Cu}_{2}$ : C, 40.66; $\mathrm{H}, 4.00 ; \mathrm{N}, 9.81 \%$.

$\left[\mathrm{Cu}_{2}(\mathrm{~L}-2)\left(\mathrm{CH}_{3} \mathrm{COO}\right)\right](3)$. This compound was prepared according to the literature. ${ }^{1 \mathrm{~b})}$

Measurements. The electronic spectra were measured with a Hitachi U-3400 spectrophotometer. The infrared spectra were obtained by $\mathrm{KBr}$-disk method with a Horiba FT-200 spectrophotometer. The magnetic susceptibilities were measured with a SQUID susceptometer of QUANTUM DESIGN MPMS Model and a Shimadzu torsion magnetometer BM-2 which were calibrated with $\mathrm{Hg}\left[\mathrm{Co}(\mathrm{NCS})_{4}\right]$. Diamagnetic correction was made by using Pascal's constants. ${ }^{18)}$ Cyclic voltammograms were obtained with a Hokuto Denko HA-301 Potentiostat/Galvanostat and a Hokuto Denko HB-104 Function Generator by using a three-electrode configuration, including a glassy carbon working electrode, a platinumcoil auxiliary electrode, and a saturated calomel electrode as a reference electrode. Acetonitrile was used as the solvent, and tetrabutylammonium perchlorate as a supporting electrolyte. X-band ESR spectra were measured on a JEOL JES-RE1X ESR spectrometer (X-band microwave unit, $100 \mathrm{kHz}$ field modulation). The band frequency was calibrated with 1,1-diphenyl-2-picrylhydrazyl (DPPH, $g=2.0036)$.

X-Ray Crystallography. A single crystal of $\mathbf{1}$ was obtained by a slow diffusion of diethyl ether into an acetonitrile solution. Since crystals were very efflorescent, a single crystal was mounted inside a glass capillary with a small amount of mother liquor to prevent a loss of water molecule from the crystal. Data were collected on a Rigaku AFC-5R four circle automated diffractometer with graphite monochromated Mo $K \alpha$ radiation $(\lambda=0.71073 \AA)$ at room temperature. Unit cell parameters were determined by a leastsquares fit to 20 reflections having $24.5^{\circ}<2 \theta<31.5^{\circ}$. Because of weak intensity data, we tried to obtain better crystals, but all the attempts were in vain. Three standard reflections were measured every 150 reflections, which show no systematic decay throughout data collection. The data were corrected for Lorentz and polariza- tion effects, and an empirical absorption correction ( $\Psi$ scans) was also carried out. Crystallographic data are summarized in Table 1. The numbers of data (3092 data) used for analysis were not enough for a satisfactory determination. Since the structure of this complex is very important in this study, we carried out the analysis. The structure was solved by the standard heavy-atom techniques. The metal atom was located by Patterson syntheses using the program SHELXS-86. ${ }^{19)}$ Full-matrix least-squares refinement and difference Fourier methods (SHELX-76) ${ }^{20)}$ were used to locate all remaining non-hydrogen atoms. The atomic scattering factors and anomalous dispersion coefficients were taken from the literatures. ${ }^{21)}$

All the non-hydrogen atoms were refined anisotropically. Hydrogen atoms were not included in the calculation. Several cycles of refinement led to convergence with $R=0.088\left(R_{\mathrm{w}}=0.085\right)$. A final difference Fourier map showed the largest peak of $0.65 \mathrm{e} \AA^{-3}$. Final atomic coordinates for the non-hydrogen atoms are given in Table 2. Table of anisotropic thermal parameters, and list of $F_{\mathrm{o}}$ and $F_{\mathrm{c}}$ are given in the supplementary materials. ${ }^{22)}$ All calculations were carried out on a FACOM M760/20 computer at the Kanazawa University Information Processing Center.

\section{Results and Discussion}

Synthesis and Characterization. $\mathrm{H}_{2} \mathrm{~L}-1$ was prepared in situ by the condensation of $\mathbf{A}$ with salicylaldehyde and used for preparation of the complex (1) without isolation. The compound $\mathbf{A}$ is very useful for preparation of a variety of unsymmetric dinucleating ligands with 1,3-diaminopropan-2-olate skeleton. For instance, we have prepared various types of unsymmetric heptadentate ligands such as $\left(\mathrm{R}_{\mathrm{a}}\right)_{2} \mathrm{NCH}_{2} \mathrm{C}(\mathrm{H}) \mathrm{OHCH}_{2} \mathrm{~N}\left(\mathrm{R}_{\mathrm{b}}\right)_{2}$, where two side arms, $\mathrm{R}_{\mathrm{a}}$ and $R_{b}$, contain a variety of donor groups. Chemistry of di- and polynuclear metal complexes of such unsymmetric dinucleating ligands are in progress.

Complexes $\mathbf{1}$ and $\mathbf{2}$ have one acetato group. In the IR spectra, $\Delta \tilde{v}\left(\mathrm{COO}^{-}\right)$values $\left(\tilde{v}_{\text {asymm }}\left(\mathrm{COO}^{-}\right)-\tilde{v}_{\text {symm }}\left(\mathrm{COO}^{-}\right)\right)$of

Table 1. Crystallographic Data for Complex 1

\begin{tabular}{ll}
\hline Formula & $\mathrm{C}_{24} \mathrm{H}_{27} \mathrm{~N}_{4} \mathrm{Cl}_{1.5} \mathrm{Cu}_{2} \mathrm{Na}_{0.5} \mathrm{O}_{11}$ \\
Fw & 739.27 \\
Space group & $C 2 / c$ \\
$a / \AA$ & $42.704(8)$ \\
$b / \AA$ & $12.689(6)$ \\
$c / \AA$ & $29.008(9)$ \\
$\beta /$ deg & $128.28(1)$ \\
$V / \AA^{3}$ & $12338(7)$ \\
$Z$ & 16 \\
Crystal size $/ \mathrm{mm}$ & $0.5 \times 0.3 \times 0.2$ \\
$\rho_{\text {calcd }} / \mathrm{g} \mathrm{cm}{ }^{-1}$ & 1.59 \\
$\lambda(\mathrm{Mo} K \alpha) / \AA$ & 0.71073 \\
$\mathrm{Scan}$ method & $\omega-2 \theta$ scan $(3<2 \theta \leq 55)$ \\
Scan speed ${ }^{\circ}$ min & \\
Max scan times & 6 \\
No. of data collected & 3 \\
No. of data used & 12060 \\
$\mu / \mathrm{cm}^{-1}$ & $3097\left(\left|F_{\mathrm{o}}\right| \geq 3.0 \sigma\left|F_{\mathrm{o}}\right|\right)$ \\
$R^{\text {a) }}$ & 6.67 \\
$R_{\mathrm{w}}{ }^{\mathrm{b})}$ & 0.088 \\
\hline
\end{tabular}

a) $R=\sum\left[\left|F_{\mathrm{o}}\right|-\left|F_{\mathrm{c}}\right| / \sum\left|F_{\mathrm{o}}\right|\right]$

b) $R_{\mathrm{w}}=\left[\sum w\left(\left|F_{\mathrm{o}}\right|-\left|F_{\mathrm{c}}\right|\right)^{2} / \sum w\left|F_{\mathrm{o}}\right|^{2}\right]^{1 / 2} ; w=4 / \sigma^{2}\left(F_{\mathrm{o}}\right)$ 
these complexes are in the range of 114 and $104 \mathrm{~cm}^{-1}$ (Table 3), respectively, suggesting that the carboxylate serves as a bridging ligand in $O, O^{\prime}-s y n-s y n$ coordination mode. ${ }^{23)}$
Structure Description of 1. The crystal structure consists of two crystallographically independent dinuclear complex cations (1a and 1b), sodium cations, perchlorate anions,

Table 2. Fractional Atomic Coordinates and Isotropic Thermal Parameters of 1 with Their Standard Deviations in Parentheses

\begin{tabular}{|c|c|c|c|c|c|c|c|c|c|}
\hline Atom & $x$ & $y$ & $z$ & $U_{\mathrm{eq}}^{\mathrm{a})}$ & Atom & $x$ & $y$ & $z$ & $U_{\mathrm{eq}}^{\text {a) }}$ \\
\hline $\mathrm{Cu} 1$ & $0.42054(7)$ & $0.8197(2)$ & $0.0364(1)$ & $0.041(1)$ & $\mathrm{C} 25$ & $0.3374(5)$ & $0.610(1)$ & $0.0256(9)$ & $0.033(8)$ \\
\hline $\mathrm{Cu} 2$ & $0.44312(7)$ & $0.8443(2)$ & $-0.0577(1)$ & $0.047(2)$ & $\mathrm{C} 26$ & $0.3532(7)$ & $0.522(2)$ & $0.018(1)$ & $0.059(9)$ \\
\hline N1 & $0.4490(4)$ & $0.696(1)$ & $0.0888(8)$ & $0.044(7)$ & $\mathrm{C} 27$ & $0.3624(6)$ & $0.524(2)$ & $-0.018(1)$ & $0.054(9)$ \\
\hline $\mathrm{N} 2$ & $0.4702(5)$ & $0.710(1)$ & $-0.0561(7)$ & $0.046(8)$ & $\mathrm{C} 28$ & $0.3569(7)$ & $0.613(2)$ & $-0.050(1)$ & $0.051(9)$ \\
\hline N3 & $0.3905(5)$ & $0.774(1)$ & $-0.1380(8)$ & $0.048(7)$ & $\mathrm{C} 29$ & $0.3417(5)$ & $0.703(1)$ & $-0.0398(9)$ & $0.033(8)$ \\
\hline N4 & $0.4802(5)$ & $0.911(1)$ & $-0.0693(7)$ & $0.039(7)$ & $\mathrm{C} 30$ & $0.3315(6)$ & $0.707(1)$ & $-0.005(1)$ & $0.047(8)$ \\
\hline O1 & $0.4418(4)$ & $0.7763(9)$ & $-0.0018(7)$ & $0.051(7)$ & C31 & $0.3277(7)$ & $0.605(2)$ & $0.065(1)$ & $0.047(9)$ \\
\hline $\mathrm{O} 2$ & $0.4212(4)$ & $0.975(1)$ & $-0.0577(7)$ & $0.067(7)$ & C32 & $0.3032(7)$ & $0.659(1)$ & $0.118(1)$ & $0.060(9)$ \\
\hline $\mathrm{O} 3$ & $0.3929(4)$ & $0.9434(9)$ & $-0.0167(6)$ & $0.041(6)$ & C33 & $0.2985(9)$ & $0.757(2)$ & $0.136(1)$ & $0.076(9)$ \\
\hline $\mathrm{O} 4$ & $0.4039(4)$ & $0.8680(9)$ & $0.0772(6)$ & $0.042(6)$ & C34 & $0.2888(9)$ & $0.774(2)$ & $0.170(1)$ & $0.084(9)$ \\
\hline $\mathrm{C} 1$ & $0.4342(6)$ & $0.735(2)$ & $0.153(1)$ & $0.056(8)$ & C35 & $0.3185(7)$ & $0.932(2)$ & $0.2372(9)$ & $0.059(9)$ \\
\hline $\mathrm{C} 2$ & $0.4405(8)$ & $0.699(2)$ & $0.201(1)$ & $0.089(9)$ & C36 & $0.3419(6)$ & $1.000(2)$ & $0.229(1)$ & $0.051(8)$ \\
\hline $\mathrm{C} 3$ & $0.4288(8)$ & $0.749(2)$ & $0.230(1)$ & $0.087(9)$ & $\mathrm{C} 37$ & $0.3820(6)$ & $1.026(2)$ & $0.280(1)$ & $0.049(8)$ \\
\hline $\mathrm{C} 4$ & $0.4076(7)$ & $0.844(2)$ & $0.203(1)$ & $0.071(9)$ & C38 & $0.4018(7)$ & $1.096(2)$ & $0.270(1)$ & $0.060(9)$ \\
\hline $\mathrm{C} 5$ & $0.3996(7)$ & $0.880(2)$ & $0.153(1)$ & $0.060(9)$ & C39 & $0.3871(7)$ & $1.132(2)$ & $0.217(1)$ & $0.067(8)$ \\
\hline C6 & $0.4121(5)$ & $0.828(1)$ & $0.124(1)$ & $0.019(8)$ & $\mathrm{C} 40$ & 0.3459 & $1.101(2)$ & $0.168(1)$ & 3(9) \\
\hline $\mathrm{C} 7$ & $0.4501(6)$ & $0.676(2)$ & $0.132(1)$ & $0.056(8)$ & $\mathrm{C} 41$ & $0.2465(6)$ & $0.893(2)$ & $0.1784(9)$ & $0.045(8)$ \\
\hline $\mathrm{C} 8$ & $0.4654(7)$ & $0.631(2)$ & $0.065(1)$ & $0.065(8)$ & C42 & $0.2304(6)$ & $1.006(2)$ & $0.156(1)$ & $0.046(9)$ \\
\hline C9 & $0.4708(7)$ & $0.694(2)$ & $0.027(1)$ & $0.073(8)$ & C43 & $0.2108(7)$ & $1.060(2)$ & $0.175(1)$ & $0.071(9)$ \\
\hline $\mathrm{C} 10$ & $0.4710(6)$ & $0.638(2)$ & $-0.017(1)$ & $0.063(8)$ & $\mathrm{C} 44$ & $0.1937(7)$ & $1.158(2)$ & $0.149(1)$ & $0.08(1)$ \\
\hline $\mathrm{C} 11$ & $0.4461(7)$ & $0.664(2)$ & $-0.115(1)$ & $0.078(9)$ & $\mathrm{C} 45$ & $0.2001(7)$ & $1.202(2)$ & $0.112(1)$ & $0.074(9)$ \\
\hline $\mathrm{C} 12$ & $0.3997(6)$ & $0.687(2)$ & $-0.1512(9)$ & $0.048(8)$ & $\mathrm{C} 46$ & $0.2201(7)$ & $1.143(2)$ & $0.099(1)$ & $0.056(9)$ \\
\hline $\mathrm{C} 13$ & $0.3725(8)$ & $0.620(2)$ & $-0.197(1)$ & $0.082(9)$ & $\mathrm{C} 47$ & $0.2650(6)$ & $1.030(1)$ & $0.012(1)$ & $0.041(8)$ \\
\hline $\mathrm{C} 14$ & $0.3325(8)$ & $0.654(2)$ & $-0.228(1)$ & $0.087(9)$ & $\mathrm{C} 48$ & $0.2526(7)$ & $1.113(1)$ & $-0.036(1)$ & $0.058(9)$ \\
\hline $\mathrm{C} 15$ & $0.3232(7)$ & $0.741(2)$ & $-0.212(1)$ & $0.071(9)$ & $\mathrm{C} 11$ & $0.2278(2)$ & $0.5160(5)$ & $0.1564(3)$ & $0.060(4)$ \\
\hline $\mathrm{C} 16$ & $0.3525(6)$ & $0.802(2)$ & $-0.166(1)$ & $0.060(9)$ & $\mathrm{O} 9$ & $0.2060(6)$ & $0.489(2)$ & $0.0953(8)$ & $0.119(9)$ \\
\hline $\mathrm{C} 17$ & $0.5099(6)$ & $0.742(1)$ & $-0.038(1)$ & $0.057(9)$ & $\mathrm{O} 10$ & $0.2683(5)$ & $0.525(1)$ & $0.178(1)$ & $0.109(8)$ \\
\hline $\mathrm{C} 18$ & $0.5067(6)$ & $0.846(1)$ & $-0.0666(8)$ & $0.038(8)$ & $\mathrm{O} 11$ & $0.2232(6)$ & $0.439(2)$ & $0.1846(8)$ & $0.120(9)$ \\
\hline C19 & $0.5282(6)$ & $0.869(2)$ & $-0.084(1)$ & $0.060(9)$ & $\mathrm{O} 12$ & $0.2167(8)$ & $0.611(2)$ & $0.165(1)$ & $0.17(1)$ \\
\hline $\mathrm{C} 20$ & $0.5252(6)$ & $0.968(2)$ & $-0.106(1)$ & $0.073(9)$ & C12 & $0.5914(2)$ & $0.6722(5)$ & $0.1469(3)$ & $0.070(4)$ \\
\hline $\mathrm{C} 21$ & $0.4979(7)$ & $1.046(2)$ & $-0.109(1)$ & $0.064(9)$ & O13 & $0.5679(7)$ & $0.610(2)$ & $0.155(1)$ & $0.178(9)$ \\
\hline $\mathrm{C} 22$ & $0.4764(6)$ & $1.008(2)$ & $-0.0907(9)$ & $0.044(8)$ & O14 & $0.5867(9)$ & $0.630(2)$ & $0.096(1)$ & $0.20(1)$ \\
\hline $\mathrm{C} 23$ & $0.3999(6)$ & $0.998(1)$ & $-0.046(1)$ & $0.040(8)$ & O15 & $0.6309(6)$ & $0.663(2)$ & $0.191(1)$ & $0.128(9)$ \\
\hline $\mathrm{C} 24$ & $0.3796(6)$ & $1.107(1)$ & $-0.064(1)$ & $0.062(8)$ & 016 & $0.5777(7)$ & $0.774(1)$ & $0.137(1)$ & $0.153(9)$ \\
\hline $\mathrm{Cu} 3$ & $0.30063(7)$ & $0.8159(2)$ & $0.0458(1)$ & $0.042(2)$ & $\mathrm{C} 13$ & $0.1248(2)$ & $0.7152(5)$ & $0.4298(3)$ & $0.074(4)$ \\
\hline $\mathrm{Cu} 4$ & $0.26929(7)$ & $0.9628(2)$ & $0.1095(1)$ & $0.043(2)$ & 017 & $0.1112(7)$ & $0.775(1)$ & $0.455(1)$ & $0.160(9)$ \\
\hline N5 & $0.3127(5)$ & $0.675(1)$ & $0.0794(9)$ & $0.049(8)$ & 018 & $0.1479(7)$ & $0.773(1)$ & $0.419(1)$ & $0.157(9)$ \\
\hline N6 & $0.2831(5)$ & $0.882(1)$ & $0.181(7)$ & $0.043(8)$ & 019 & $0.144(1)$ & $0.629(2)$ & $0.463(2)$ & $0.27(1)$ \\
\hline N7 & $0.3272(5)$ & $1.035(1)$ & $0.1770(8)$ & $0.048(8)$ & $\mathrm{O} 20$ & $0.0930(8)$ & $0.655(2)$ & $0.379(1)$ & $0.17(1)$ \\
\hline N8 & $0.2355(5)$ & $1.047(1)$ & $0.1198(8)$ & $0.047(8)$ & Ow1 & $0.486(1)$ & $0.392(3)$ & $0.163(2)$ & $0.34(1)$ \\
\hline O5 & $0.2851(4)$ & $0.835(1)$ & $0.092(7)$ & $0.056(7)$ & Ow $2^{\text {b) }}$ & 0.000 & $0.588(5)$ & 0.250 & $0.39(1)$ \\
\hline O6 & $0.2541(4)$ & $1.038(1)$ & $0.0418(7)$ & $0.064(7)$ & Ow $3^{\text {b) }}$ & $0.407(3)$ & $0.430(6)$ & $0.213(5)$ & $0.40(1)$ \\
\hline O7 & $0.2892(4)$ & $0.960(1)$ & $0.0151(6)$ & $0.052(7)$ & $\mathrm{Na}$ & $0.3423(2)$ & $0.9613(5)$ & $0.0014(4)$ & $0.045(4)$ \\
\hline $\mathrm{O} 8$ & $0.3164(4)$ & $0.7928(9)$ & $0.0000(6)$ & $0.039(7)$ & & & & & \\
\hline
\end{tabular}

a) $U_{\mathrm{eq}}=1 / 3\left(U_{11}+U_{22}+U_{33}\right)$. b) The occupancy factor of the atom is 0.5 .

Table 3. Electronic and IR Spectral Data of 1, 2, and 3

\begin{tabular}{|c|c|c|c|}
\hline \multirow[t]{2}{*}{ Complex } & \multicolumn{2}{|c|}{ Absorption band maxima $^{\text {a) }}$} & \multirow{2}{*}{$\frac{\tilde{v}_{\text {asymm }}\left(\mathrm{COO}^{-}\right)-\tilde{\nu}_{\text {symm }}\left(\mathrm{COO}^{-}\right)}{\tilde{v} / \mathrm{cm}^{-1}}$} \\
\hline & \multicolumn{2}{|c|}{$\tilde{v} / \mathrm{cm}^{-1)} /\left(\varepsilon / \mathrm{mol}^{-1} \mathrm{dm}^{3} \mathrm{~cm}^{-1}\right)$} & \\
\hline 1 & $11000^{\mathrm{s}}$ & $15900(231)$ & 114 \\
\hline 2 & $11100(185)$ & $14500^{\mathrm{s}}$ & 104 \\
\hline 3 & & 15900 (396) & 111 \\
\hline
\end{tabular}


and lattice waters. $\mathbf{1 a}$ and $\mathbf{1 b}$ are geometrical isomers with a different configuration of an asymmetric carbon atom of L1. Two dinuclear cations are linked by sodium cation which is located in a distorted octahedron formed by oxygen atoms of phenolates, bridging carboxylates, and perchlorates. The $\mathrm{Na}-\mathrm{O}$ contacts are in the range of $2.3-2.6 \AA$.

Molecular structures of $\mathbf{1 a}$ and $\mathbf{1 b}$ are shown in Fig. 1 and the selected bond distances and angles are given in Table 4. 1a and $\mathbf{1 b}$ consist of both square planar and distorted square pyramidal sites, in which two copper atoms are doubly bridged by endogenous alkoxide and exogenous

Table 4. Selected Bond Distances $(l / \AA)$ and Angles $\left({ }^{\circ}\right)$ of $\mathbf{1}$

\begin{tabular}{|c|c|c|c|}
\hline \multicolumn{4}{|c|}{ Distances } \\
\hline \multicolumn{2}{|c|}{ Molecule 1a } & \multicolumn{2}{|c|}{ Molecule 1b } \\
\hline $\mathrm{Cu} 1-\mathrm{N} 1$ & $1.99(1)$ & $\mathrm{Cu} 3-\mathrm{N} 5$ & $1.94(2)$ \\
\hline $\mathrm{Cu} 1-\mathrm{O} 1$ & $1.90(2)$ & $\mathrm{Cu} 3-\mathrm{O} 5$ & $1.84(2)$ \\
\hline $\mathrm{Cu} 1-\mathrm{O} 3$ & $1.99(1)$ & $\mathrm{Cu} 3-\mathrm{O} 7$ & $1.96(1)$ \\
\hline $\mathrm{Cu} 1-\mathrm{O} 4$ & $1.83(2)$ & $\mathrm{Cu} 3-\mathrm{O} 8$ & $1.85(2)$ \\
\hline $\mathrm{Cu} 2-\mathrm{N} 2$ & $2.05(2)$ & $\mathrm{Cu} 4-\mathrm{N} 6$ & $2.05(2)$ \\
\hline $\mathrm{Cu} 2-\mathrm{N} 3$ & 2.19(1) & $\mathrm{Cu} 4-\mathrm{N} 7$ & $2.19(1)$ \\
\hline $\mathrm{Cu} 2-\mathrm{N} 4$ & $1.99(2)$ & $\mathrm{Cu} 4-\mathrm{N} 8$ & $1.96(2)$ \\
\hline $\mathrm{Cu} 2-\mathrm{O} 1$ & $1.87(2)$ & $\mathrm{Cu} 4-\mathrm{O} 5$ & $1.95(2)$ \\
\hline $\mathrm{Cu} 2-\mathrm{O} 2$ & $1.90(2)$ & $\mathrm{Cu} 4-\mathrm{O} 6$ & $1.90(2)$ \\
\hline $\mathrm{Cu} 1-\mathrm{Cu} 2$ & $3.426(6)$ & $\mathrm{Cu} 3-\mathrm{Cu} 4$ & $3.428(5)$ \\
\hline \multicolumn{4}{|c|}{ Angles } \\
\hline \multicolumn{2}{|c|}{ Molecule 1a } & \multicolumn{2}{|c|}{ Molecule 1b } \\
\hline $\mathrm{N} 1-\mathrm{Cu} 1-\mathrm{O} 1$ & $86.0(8)$ & N5-Cu3-O5 & $81.9(9)$ \\
\hline $\mathrm{N} 1-\mathrm{Cu} 1-\mathrm{O} 3$ & $178.7(6)$ & N5-Cu3-O7 & $177.6(7)$ \\
\hline $\mathrm{N} 1-\mathrm{Cu} 1-\mathrm{O} 4$ & $93.9(8)$ & $\mathrm{N} 5-\mathrm{Cu} 3-\mathrm{O} 8$ & $96.5(8)$ \\
\hline $\mathrm{O} 1-\mathrm{Cu} 1-\mathrm{O} 3$ & $92.7(7)$ & $\mathrm{O} 5-\mathrm{Cu} 3-\mathrm{O} 7$ & $96.3(8)$ \\
\hline $\mathrm{O} 1-\mathrm{Cu} 1-\mathrm{O} 4$ & $175.2(9)$ & $\mathrm{O} 5-\mathrm{Cu} 3-\mathrm{O} 8$ & $179(1)$ \\
\hline $\mathrm{O} 3-\mathrm{Cu} 1-\mathrm{O} 4$ & 87.3(7) & $\mathrm{O} 7-\mathrm{Cu} 3-\mathrm{O} 8$ & $85.4(8)$ \\
\hline $\mathrm{N} 2-\mathrm{Cu} 2-\mathrm{N} 3$ & $83.0(6)$ & $\mathrm{N} 6-\mathrm{Cu} 4-\mathrm{N} 7$ & $81.3(7)$ \\
\hline $\mathrm{N} 2-\mathrm{Cu} 2-\mathrm{N} 4$ & $81.9(8)$ & $\mathrm{N} 6-\mathrm{Cu} 4-\mathrm{N} 8$ & $83.9(9)$ \\
\hline $\mathrm{N} 2-\mathrm{Cu} 2-\mathrm{O} 1$ & $85.1(8)$ & N6-Cu4-O5 & $85.4(7)$ \\
\hline $\mathrm{N} 2-\mathrm{Cu} 2-\mathrm{O} 2$ & $176.0(7)$ & $\mathrm{N} 6-\mathrm{Cu} 4-\mathrm{O} 6$ & $177.5(8)$ \\
\hline $\mathrm{N} 3-\mathrm{Cu} 2-\mathrm{N} 4$ & $113.1(8)$ & $\mathrm{N} 7-\mathrm{Cu} 4-\mathrm{N} 8$ & $99.8(8)$ \\
\hline $\mathrm{N} 3-\mathrm{Cu} 2-\mathrm{O} 1$ & 99.9(7) & N7-Cu4-O5 & $101.5(6)$ \\
\hline $\mathrm{N} 3-\mathrm{Cu} 2-\mathrm{O} 2$ & $100.0(6)$ & $\mathrm{N} 7-\mathrm{Cu} 4-\mathrm{O} 6$ & $100.8(7)$ \\
\hline $\mathrm{N} 4-\mathrm{Cu} 2-\mathrm{O} 1$ & $142.5(9)$ & N8-Cu4-O5 & $154.4(8)$ \\
\hline $\mathrm{N} 4-\mathrm{Cu} 2-\mathrm{O} 2$ & $94.4(8)$ & $\mathrm{N} 8-\mathrm{Cu} 4-\mathrm{O} 6$ & 94.3(9) \\
\hline $\mathrm{O} 1-\mathrm{Cu} 2-\mathrm{O} 2$ & $97.0(8)$ & $\mathrm{O} 5-\mathrm{Cu} 4-\mathrm{O} 6$ & $95.5(7)$ \\
\hline $\mathrm{Cu} 1-\mathrm{O} 1-\mathrm{Cu} 2$ & $130.9(7)$ & $\mathrm{Cu} 3-\mathrm{O} 5-\mathrm{Cu} 4$ & $129.8(8)$ \\
\hline
\end{tabular}

acetate oxygens. The $\mathrm{Cu} \cdot \mathrm{Cu}$ separations of $\mathbf{1 a}$ and $\mathbf{1 b}$ are 3.426(6) $\AA$ and 3.428(5) $\AA$, respectively, and the $\mathrm{Cu}-\mathrm{O}-$ (alkoxo)-Cu angles are $130.9(7)$ and $129.8(8)^{\circ}$, respectively. These values are comparable to those of the parent complex $3^{11 b)}\left(3.495(3) \AA\right.$ and $\left.134.5^{\circ}\right)$ and a closely related complex $\left[\mathrm{Cu}_{2}(\mathrm{~L}-\mathrm{Et})\left(\mathrm{CH}_{3} \mathrm{COO}\right)\right]^{2+}(\mathbf{4})^{10 \mathrm{~b})}\left(3.459(2) \AA\right.$ and $\left.130.6(5)^{\circ}\right)$ which has 2-benzimidazolylmethyl side arms instead of 2pyridylmethyl side arms. Stereochemistries of both five-coordinate moieties in $\mathbf{1 a}$ and $\mathbf{1 b}$ are best described as distorted square pyramidal structure. This is in contrast with those found for $\mathbf{4}$ in which both five-coordinate copper moieties have slightly distorted trigonal bipyramidal structure. ${ }^{10 b)}$ The $\mathrm{O} 1, \mathrm{O} 2, \mathrm{~N} 2$, and $\mathrm{N} 4$ atoms in $1 \mathrm{a}$ and the O5, O6, N6, and N8 atoms in $\mathbf{1 b}$ form basal planes. The apical bond distances of Cu2-N3 and Cu4-N7 (2.19(1) and 2.19(1) $\AA$ ) are clearly longer than the basal bond distances of $\mathrm{Cu}-\mathrm{N}(1.96(2)-$ 2.05(2) $\AA$ ). Distortions from square pyramidal structure in 1a and 1b become clear by comparing the angles around copper atoms. The $\mathrm{N} 2-\mathrm{Cu} 2-\mathrm{O} 2$ and $\mathrm{N} 6-\mathrm{Cu} 4-\mathrm{O} 6$ axis are almost linear $\left(176.0(7)\right.$ and $\left.177.5(8)^{\circ}\right)$. The N4-Cu2-O1, $\mathrm{N} 3-\mathrm{Cu} 2-\mathrm{O} 1$, and N3-Cu2-N4 angles in 1a are 142.5(9), 99.9(7), and $113.1(8)^{\circ}$, respectively, while the N8-Cu4-O5, $\mathrm{N} 7-\mathrm{Cu} 4-\mathrm{O} 5$, and N7-Cu4-N8 angles in $\mathbf{1 b}$ are 154.4(8), 101.5(6), and $99.8(8)^{\circ}$, indicating that distortion from a square pyramidal structure in $\mathbf{1 a}$ is more pronounced than that in 1b. There is a difference between conformations of the five-membered chelate rings of $\mathrm{O}($ alkoxo $)-\mathrm{CR}(\mathrm{H})-\mathrm{C}-$ $\left(\mathrm{H}_{2}\right)-\mathrm{N}$ (tertiary amine) in $\mathbf{1 a}$ and $\mathbf{1 b}$ as shown in Fig. 2. Such conformational difference seems to be closely related to a degree of distortion from a square pyramidal structure.

Bond distances and angles of square planar moieties are similar to those of 3 except for the N5-Cu3-O5 angle $\left(81.9(9)^{\circ}\right)$ in $\mathbf{1 b}$, which is smaller than those of $\mathbf{1 a}$ and $\mathbf{3}$ $\left(86.0(8)\right.$ ) and $\left.86.5(4)^{\circ}\right)$. This may be due to a conformational effect of five-membered bridging chelate ring.

Electronic Spectra. The electronic spectra of $\left[\mathrm{Cu}_{2}(\mathrm{~L}-1)-\right.$ $\left.\left(\mathrm{CH}_{3} \mathrm{COO}\right)\right]^{+}(\mathbf{1}),\left[\mathrm{Cu}_{2}(\mathrm{tpdp})\left(\mathrm{CH}_{3} \mathrm{COO}\right)\right]^{2+}(\mathbf{2})$, and $\left[\mathrm{Cu}_{2}(\mathrm{~L}-\right.$ 2) $\left.\left(\mathrm{CH}_{3} \mathrm{COO}\right)\right](3)^{11 \mathrm{~b})}$ in acetonitrile are shown in Fig. 3 (Table 3 ). The spectrum of 1 shows a $d-d$ band at 15900 $\mathrm{cm}^{-1}\left(\varepsilon=231 \mathrm{~mol}^{-1} \mathrm{dm}^{3} \mathrm{~cm}^{-1}\right)$ with a shoulder at ca. $11000 \mathrm{~cm}^{-1}$. This spectral feature is well interpreted in terms of superposition of the $\mathrm{d}-\mathrm{d}$ bands of the parent complexes $\mathbf{2}$ and $\mathbf{3}$, i.e. $\mathbf{3}$ which has two square planar cop-

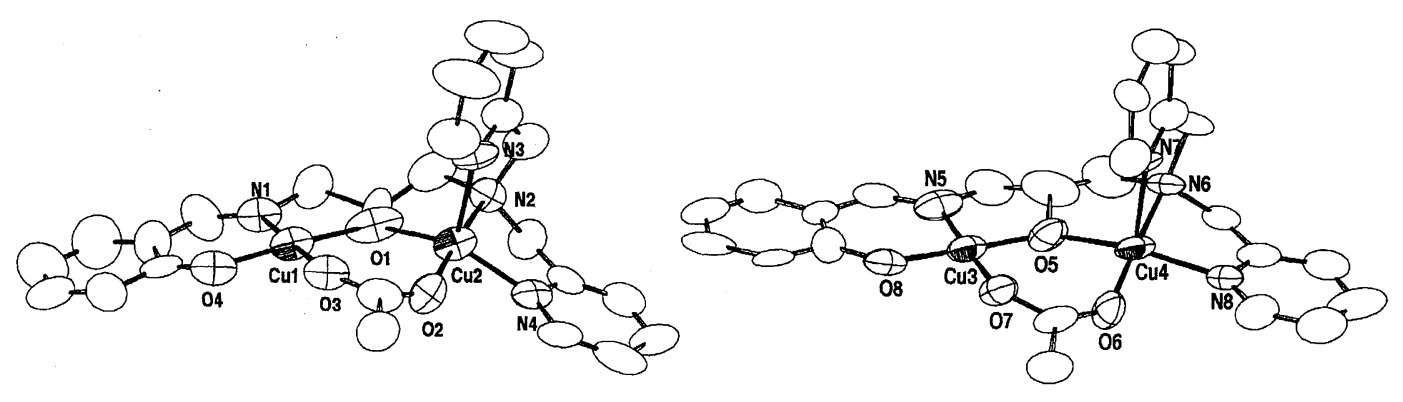

$1 a$

Fig. 1. Molecular structures of complex cations $\mathbf{1 a}$ and $\mathbf{1 b}$. 


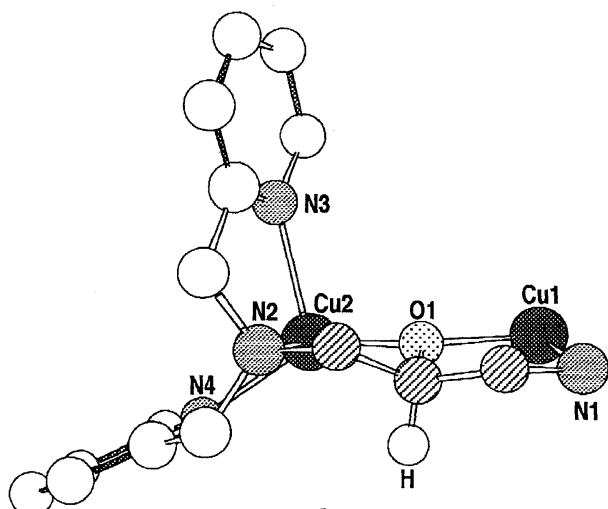

$1 a$

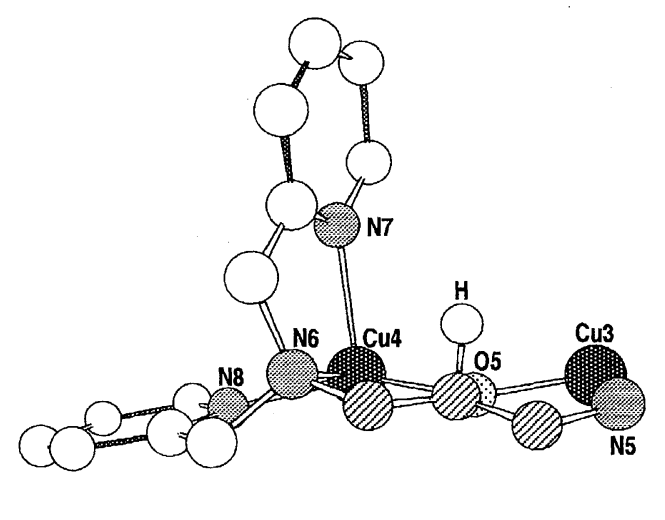

$1 b$

Fig. 2. Conformations of the bridging chelate rings of $\mathbf{1 a}$ and $\mathbf{1 b}$.

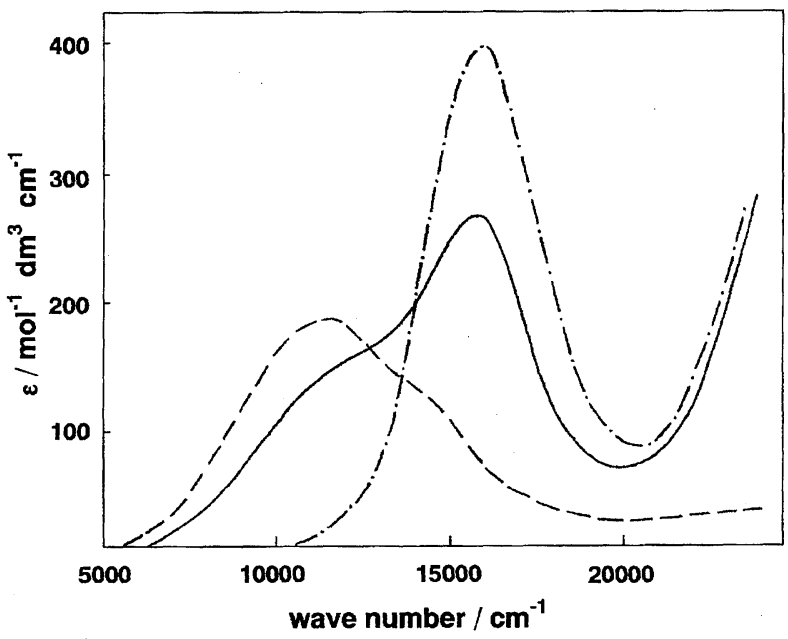

Fig. 3. Electronic spectra of $\mathbf{1}(-), \mathbf{2}(--)$, and $\mathbf{3}(-\cdot-)$ in acetonitrile.

per sites ${ }^{11 \mathrm{~b})}$ exhibits a $\mathrm{d}-\mathrm{d}$ band maximum at $15900 \mathrm{~cm}^{-1}$ $\left(\varepsilon=396 \mathrm{~mol}^{-1} \mathrm{dm}^{3} \mathrm{~cm}^{-1}\right)$ and 2 at $11100 \mathrm{~cm}^{-1}(\varepsilon=185$ $\mathrm{mol}^{-1} \mathrm{dm}^{3} \mathrm{~cm}^{-1}$ ) with a high energy shoulder at ca. 14100 $\mathrm{cm}^{-1}$. A high energy shoulder observed in $\mathbf{2}$ is characteristic of the spectral pattern of trigonal bipyramidal complexes, suggesting that the stereochemistry of five-coordinate copper centers is trigonal bipyramidal in acetonitrile. As mentioned above, a closely related complex $\mathbf{4}$ has a trigonal bipyramidal structure in solid state. ${ }^{10 b)}$

ESR Spectra. ESR spectrum of a frozen solution sample of $\mathbf{1}$ in acetonitrile saturated with tetrabutylammonium perchlorate at $77 \mathrm{~K}$ is given in Fig. 4. The spectrum shows two signals at $g=4.5$ and $g=\mathrm{ca}$. 2. The former one corresponds to $\Delta M_{\mathrm{s}}=2$ transitions and the latter corresponds to $\Delta M_{\mathrm{s}}=1$ transitions with $g_{\|}=2.22$ and $g_{\perp}=2.06$. In the $g_{\|}$region, four hyperfine lines are observed with an average $A_{\|}(\mathrm{Cu})=165$ gauss. There is no evidence for zero field splitting. Although there are two structurally distinct species in solid state as mention above, the spectrum suggests that only one species is present in solution state. A powdered sample of $\mathbf{1}$ also showed a similar ESR spectrum to that of the frozen solution sample at $77 \mathrm{~K}$ with $g=4.5\left(\Delta M_{\mathrm{s}}=2\right)$ and $g=$ ca. $2\left(\Delta M_{\mathrm{s}}=1\right.$;

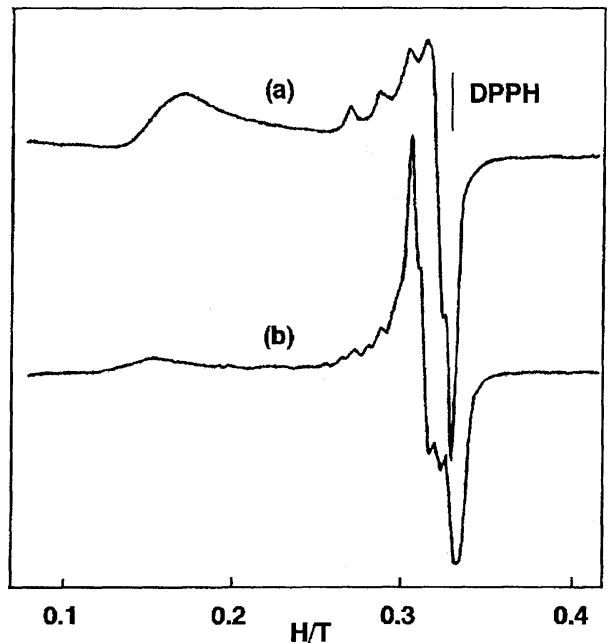

Fig. 4. ESR spectra of frozen solution samples of $\mathbf{1}$ (a) and $\mathbf{2}$ (b) in acetonitrile saturated with tetrabutylammonium perchlorate at $78 \mathrm{~K}$.

$g_{\|}=2.26$ and $\left.g_{\perp}=2.06\right)$. The average $g$ value (2.12) of a powdered sample was used for analysis of the magnetism of 1 (vide infra). The observation of only a single ESR signal for a powdered sample suggests the presence of some interaction such as dipole-dipole interaction between two dimers, $\mathbf{1 a}$ and $\mathbf{1 b}$, which are linked with $\mathrm{Na}^{+}$cation and the separation of the $\mathrm{Cu} 1$ in $\mathbf{1 a}$ and $\mathrm{Cu} 3$ in $\mathbf{1 b}$ is $5.3 \AA$.

ESR spectrum of a frozen solution sample of $\mathbf{2}$ in acetonitrile containing tetrabutylammonium perchlorate at $78 \mathrm{~K}$ also shows $\Delta M_{\mathrm{s}}=2$ transitions in half-field region and $\Delta M_{\mathrm{s}}=1$ transitions in $g=2$ region (Fig. 4). Small zero field splitting is observed and seven hyperfine lines are also observed in $g=2$ region. For many dinuclear copper(II,II) complexes, such seven hyperfine lines have been observed due to coupling of the unpaired electrons with two copper ions. ${ }^{24)}$ Only four hyperfine lines found for $\mathbf{1}$ implies that the electrons are localized at each copper site within ESR time scale.

Electrochemistry. The cyclic voltammograms (CV) of the complexes 1, 2, and 3 are shown in Fig. 5. The CV of 3 exhibits two reduction waves at -0.95 and $-1.13 \mathrm{~V}$ vs. SCE, which appear to correspond to $\mathrm{Cu}_{2}(\mathrm{II}, \mathrm{II}) / \mathrm{Cu}_{2}(\mathrm{II}, \mathrm{I})$ 


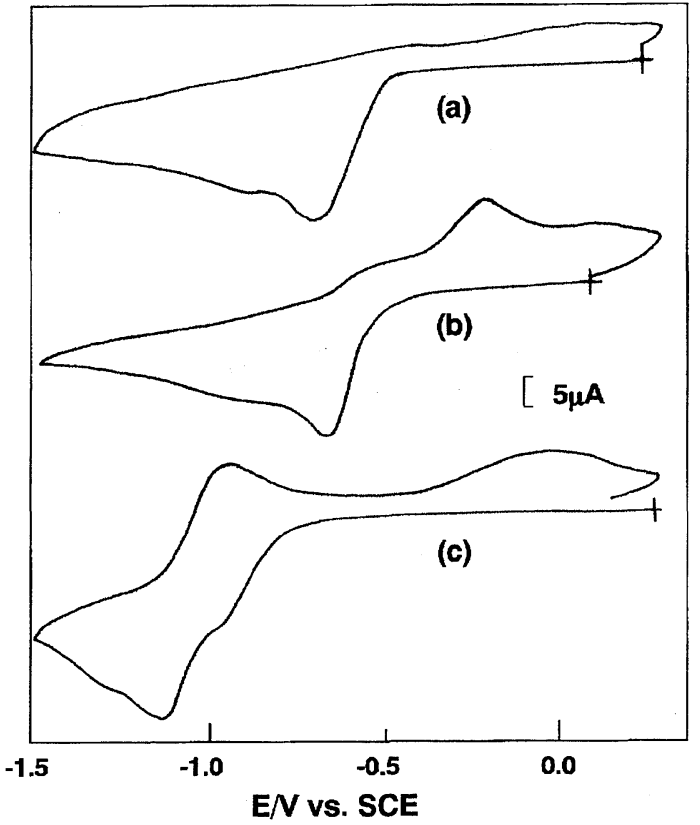

Fig. 5. Cyclic voltammograms of $\mathbf{1}(\mathbf{a}), \mathbf{2}(\mathbf{b})$, and $\mathbf{3}$ (c) in acetonitrile containing $\left(0.1 \mathrm{~mol} \mathrm{dm}^{-3}\right.$ tetrabutylammonium perchlorate) at a glassy carbon electrode with scan rate 100 $\mathrm{mV} \mathrm{s}^{-1}$.

and $\mathrm{Cu}_{2}(\mathrm{II}, \mathrm{I}) / \mathrm{Cu}_{2}(\mathrm{I}, \mathrm{I})$ reductions. The $\mathrm{CV}$ of 2 shows only one reduction wave at $-0.67 \mathrm{~V}$ vs. SCE and no further clear reduction wave is detected. The $\mathrm{CV}$ of 1 resembles that of 2. A reduction wave at $-0.68 \mathrm{~V}$ vs. SCE is almost the same as that of 2 . This indicates that the square planar sites have no significant influence on the redox chemistry of fivecoordinate sites. However, reduction of the square planar sites is not detected as found for 3 . The five-coordinated sites affect the redox chemistry of square-planar sites.

Magnetic Properties. The magnetic susceptibility of a powdered sample of $\mathbf{1}$ was measured in the temperature range $300-5 \mathrm{~K}$ (Fig. 6). The effective magnetic moment (2.60 B.M. $\left./ \mathrm{Cu}_{2}\right)$ is almost constant over the temperature range $300-60 \mathrm{~K}$, whereas it decreases to $1.81 \mathrm{~B} . \mathrm{M}$. at 5 $\mathrm{K}$, indicating the presence of antiferromagnetic interaction between copper ions. Magnetic susceptibility is given by Bleaney-Bowers equation for the isotropic exchange interaction $\left(H=-2 J S_{1} \cdot S_{2}\right)$. Since there are two structurally different species in solid state, two pairs of $g_{1}$ and $J_{1}$, and $g_{2}$ and $J_{2}$ were introduced as follows:

$$
\begin{aligned}
X_{m}\left(\mathrm{Cu}_{2}\right)= & \frac{N g_{1}{ }^{2} \beta^{2}}{k T} \cdot \frac{1}{3+\exp \left(-2 J_{1} / k T\right)} \\
& +\frac{N g_{2}{ }^{2} \beta^{2}}{k T} \cdot \frac{1}{3+\exp \left(-2 J_{2} / k T\right)}+N_{\alpha}
\end{aligned}
$$

where the symbols have their usual meanings and $N_{\alpha}$ is fixed as $120 \times 10^{-6}$ emu mole ${ }^{-1}$. Two different approaches were carried out to fit the data. First, all $g_{1}$ and $g_{2}$, and $J_{1}$ and $J_{2}$ were varied as parameters. However, reasonable $g_{1}$ and $g_{2}$ values could not be obtained by least squares fitting. Then, $g_{1}$ and $g_{2}$ were fixed as 2.12 which was assumed from $g$-value obtained from ESR spectrum of a powdered sample. A good fit to the experimental data was obtained with $J_{1}=-0.18$ $\mathrm{cm}^{-1}$ and $J_{2}=-9.05 \mathrm{~cm}^{-1}$. The theoretical curve is given as a solid line in Fig. 6. It was also attempted to fit the data by assuming that magnetic interactions in two isomers are the same $\left(J_{1}=J_{2}\right) . J\left(J_{1}=J_{2}\right)$ value obtained is $-2.90 \mathrm{~cm}^{-1}$. However, fitting is much better for the former case using two different $J_{1}$ and $J_{2}$ values (Fig. 6 insert). It seems likely that

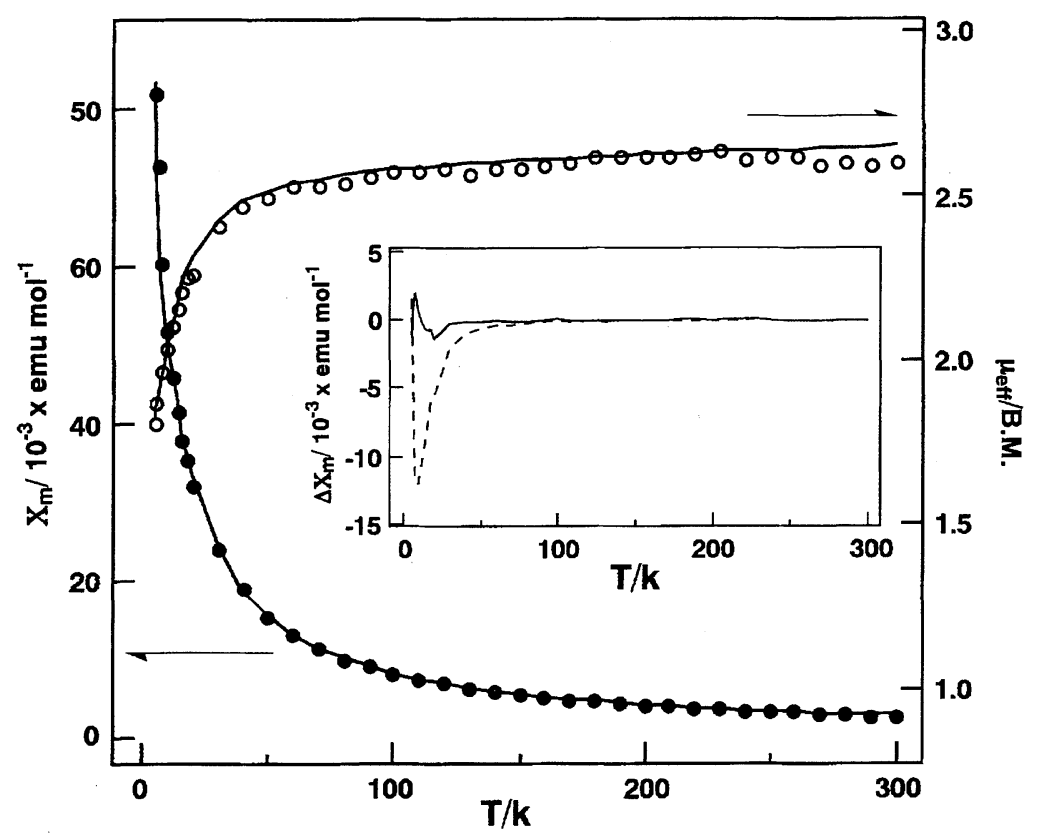

Fig. 6. Temperature dependencies of magnetic susceptibilities and magnetic moments of $\mathbf{1}$. The solid lines are the best fit curves with the parameters, $J_{1}=-9.05 \mathrm{~cm}^{-1}, J_{2}=-0.18 \mathrm{~cm}^{-1}, g=2.12$, and $T I P=1.2 \times 10^{-4} \mathrm{emu} \mathrm{mol}^{-1}$. The insert is a comparison of $\Delta X_{\mathrm{m}}\left(X_{\text {measured }}-X_{\text {calculated }}\right)$, where the solid line is $\Delta X_{\mathrm{m}}\left(X_{\text {measured }}-X_{\text {calculated }}\right)$ calculated by using two $J_{1}$ and $J_{2}$ values, and the dotted line is that calculated by using $J=-2.90 \mathrm{~cm}^{-1}$ (see text). 
two species exert different $J$ values (ca. $-0.18 \mathrm{~cm}^{-1}$ and $-9.05 \mathrm{~cm}^{-1}$ ), since magnetic interactions between copper(II) ions are highly dependent on structural characteristics. In either case, antiferromagnetic interaction(s) is relatively weak with the range of $J=0--9 \mathrm{~cm}^{-1}$.

Nishida and Kida reported that $\mathbf{3}$ shows moderately strong antiferromagnetic interaction $\left(J=-85 \mathrm{~cm}^{-1}\right) .{ }^{1 \text { b) }} J$ values of the closely related complexes with a Schiff base ligand containing 1,3-diamino-2-propanolate bridging skeleton are comparable to that of $\mathbf{3}$. However, $\mathbf{1 a}$ and $\mathbf{1 b}$ show much weaker antiferromagnetic interaction(s). In the square pyramidal moieties of $\mathbf{1 a}$ and $\mathbf{1 b}$, unpaired electrons reside in the $d_{x^{2}-y^{2}}$ orbitals whose lobes are almost directed toward both bridging ligands (alkoxo and acetato bridges) as those in the square planar moieties. Comparison of magnetic and structural features of $\mathbf{1 a}, \mathbf{1 b}, \mathbf{3}$, and $\mathbf{4}$ is given in Table 5 . There is no remarkable structural difference in bridging units between 1a, 1b, and 3. According to Hoffmann et al. ${ }^{25)}$ and Kahn et al., ${ }^{26)}$ magnetic exchange interaction is dependent on the interaction between two metal magnetic orbitals through bridging ligand(s) and the resulting energy gap between HOMO and LUMO. For further elucidation of the magnetisms of the present complexes, it is useful to know the nature of HOMO and LUMO. We have carried out EHMO calculation. ${ }^{27-29)}$

The calculation models are $\left[\mathrm{Cu}(\mathrm{OH})\left(\mathrm{NH}_{3}\right)(\mu-\mathrm{OH})(\mu\right.$ $\left.\mathrm{HCOO})\left(\mathrm{NH}_{3}\right)_{3} \mathrm{Cu}\right]^{+}\left(\mathbf{1} \mathbf{a}^{\prime}\right.$ and $\left.\mathbf{1 b}^{\prime}\right)$ for $\mathbf{1 a}$ and $\mathbf{1 b}$, and $[\mathrm{Cu}-$ $\left.(\mathrm{OH})\left(\mathrm{NH}_{3}\right)(\mu-\mathrm{OH})(\mu-\mathrm{HCOO})(\mathrm{OH})\left(\mathrm{NH}_{3}\right) \mathrm{Cu}\right]\left(3^{\prime}\right)$ for 3 , respectively, and their structural parameters are taken from $\mathrm{X}$ ray structures given in Table 4 and Ref. $11 \mathrm{~b}$. The results of calculations for $\mathbf{1 a}^{\prime}$ and $\mathbf{1} \mathbf{b}^{\prime}$ reveal that the nature of HOMO and LUMO of these two species significantly differs: The energy gap between HOMO and LUMO in $\mathbf{1 b}^{\prime}(0.186 \mathrm{eV})$ is substantially larger than that of $\mathbf{1 a}^{\prime}(0.006 \mathrm{eV})$ as seen in Fig. 7. In addition, the HOMO in $\mathbf{1 b}^{\prime}$ is solely composed of the $d_{x^{2}-y^{2}}$ orbital of the square pyramidal site and the LUMO from the $d_{x^{2}-y^{2}}$ orbital of the square planar site, whereas those in $\mathbf{1 a}^{\prime}$ are almost equally composed of $d_{x^{2}-y^{2}}$ orbitals of both sites. This would verify the use of different $J$ values in analysis of magnetisms for $\mathbf{1 a}$ and $\mathbf{1 b}$.

The observed weak antiferromagnetic interactions in 1a and $\mathbf{1 b}$ compared with that of $\mathbf{3}$ may be qualitatively interpreted in terms of degree of mixing of two magnetic orbitals

Table 5. Comparison of Magnetic and Structural Features of $\mathbf{1}, \mathbf{2}, \mathbf{3},{ }^{1 \mathrm{~b})}$ and $\mathbf{4}^{10 \mathrm{~b})}$

\begin{tabular}{lcccc}
\hline Complex & $\mathbf{1}$ & $\mathbf{2}$ & $\mathbf{3}$ & $\mathbf{4}$ \\
\hline$J / \mathrm{cm}^{-1}$ & $-0--9$ & -1.18 & -85 & $\theta=9 \mathrm{~K}$ \\
$r(\mathrm{Cu} \cdots \mathrm{Cu}) / \AA$ & 3.43 & & 3.50 & 3.46 \\
$\mathrm{Cu}-\left(\mathrm{O}_{\text {alkoxo }}\right)-\mathrm{Cu} / \AA$ & $130.9,129.8$ & & 135 & 131 \\
Solid angle around $\mathrm{O}_{\text {alkoxo }} /{ }^{\circ}$ a) & 357,356 & & 358.1 & \\
\hline
\end{tabular}

a) Summation of the three angles around $\mathrm{O}_{\text {alkoxo }}, \angle \mathrm{Cu}(1$ or 3$)-\mathrm{O}_{\text {alkoxo }}-\mathrm{Cu}(2$ or 4$), \angle \mathrm{Cu}(1$ or 3) $-\mathrm{O}_{\text {alkoxo }}-\mathrm{C}_{\text {asym }}$, and $\angle \mathrm{Cu}(2$ or 4$)-\mathrm{O}_{\text {alkoxo }}-\mathrm{C}_{\text {asym }}$.

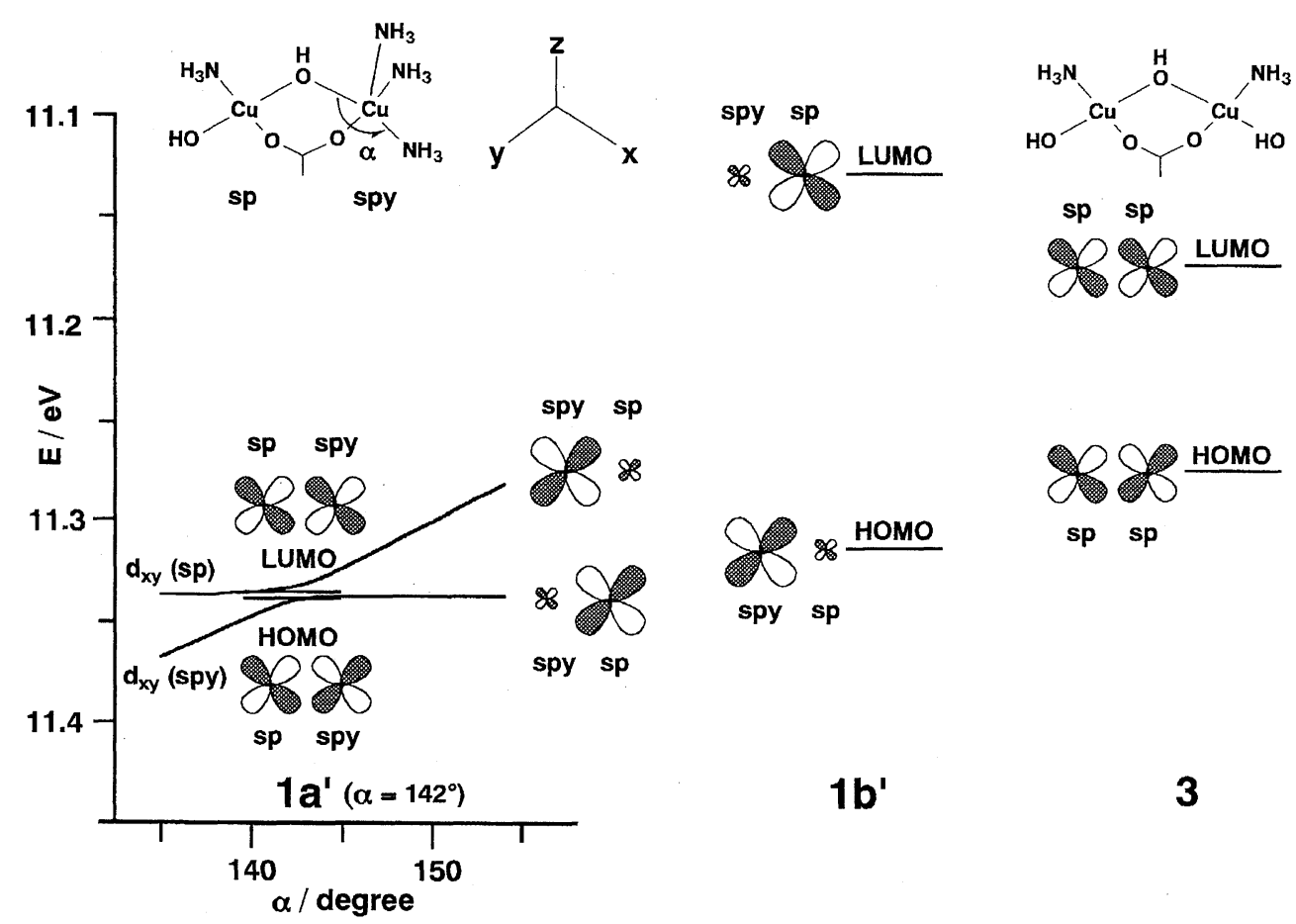

Fig. 7. Energies of HOMO and LUMO for $\mathbf{1 a}^{\prime}$ and $\mathbf{1 b}^{\prime}$, and for deformation $(\alpha)$ of $\mathbf{1 a}^{\prime}$, where sp and spy represent square planar and square pyramidal site, respectively. The variable $\alpha$ is defined as above. 
and the energy gap between HOMO and LUMO. Although the energy gap $(0.186 \mathrm{eV})$ in $\mathbf{1} \mathbf{b}^{\prime}$ is larger than that of $\mathbf{3}(0.103$ $\mathrm{eV}$ ), mixing of two magnetic $d_{x^{2}-y^{2}}$ orbitals in $\mathbf{1 b ^ { \prime }}$ is negligibly small as mentioned above. This suggests that there is a significant energy difference between two magnetic $d_{x^{2}-y^{2}}$ orbitals in square planar and distorted square pyramidal sites, ressulted in a smaller overlap between those two orbitals and a weak magnetic exchange interaction. A similar observation was also made for $\left[\mathrm{Cu}(\mathrm{en})_{2}\right]\left[\mathrm{Cu}(\mathrm{ox})_{2}\right]^{30}{ }^{30}$

On the other hand, the energy gap $(0.006 \mathrm{eV})$ between HOMO and LUMO in $\mathbf{1 a}^{\prime}$ is significantly small, which seems to be responsible for the observed weak antiferromagnetic interaction in 1a. Figure 7 shows energies of HOMO and LUMO for deformation of $\mathbf{1 a}^{\prime}$ where deformation from the original structure to square pyramidal and trigonal pyramidal structure leads to larger energy gap between HOMO and LUMO. However, as the energy gap becomes larger, mixing of two magnetic orbitals becomes smaller. This situation suggests that deformation to square planar and trigonal pyramidal structure would not significantly modulate the magnetism.

The effective magnetic moment of 2 at $300 \mathrm{~K}$ is 2.81 B.M. $/ \mathrm{Cu}_{2}$ and gradually decreases $2.54 \mathrm{~B} . \mathrm{M} . / \mathrm{Cu}_{2}$ at $10 \mathrm{~K}$, indicating the presence of very weak antiferromagnetic interaction $\left(J=-1.18 \mathrm{~cm}^{-1}\right.$ and $\left.g=2.19\right)$. The complex $4,\left[\mathrm{Cu}_{2}(\mathrm{~L}-\right.$ $\left.\mathrm{Et})\left(\mathrm{CH}_{3} \mathrm{COO}\right)\right]^{2+}$, shows a very weak ferromagnetic interaction $(\theta=9 \mathrm{~K})$. Magnetic interactions for both complexes are very weak.

Summary. A novel unsymmetric dinucleating ligand which can provide donor atom, coordination number, and geometric asymmetries at metal centers was synthesized. It formed a dicopper(II) complex containing an exogenous acetate bridge which has one square-planar site with a $\mathrm{NO}_{3}$ donor set and one five-coordinate site with a $\mathrm{N}_{3} \mathrm{O}_{2}$ donor set. The complex allows to compare its physicochemical properties with those of the parent complexes; the electronic spectral feature in the $\mathrm{d}-\mathrm{d}$ region was interpreted in terms of superposition of the $\mathrm{d}-\mathrm{d}$ bands of the parent complexes, while the magnetism and electrochemistry were modulated more or less due to the asymmetric nature of coordination environments.

We wish to thank Professor Takesi Sakurai of Kanazawa University for allowing us to use the ESR spectrometer. The present study was partially supported by a Grant-in-Aid for Scientific Research by the Ministry of Education, Science and Culture.

\section{References}

1) P. A. Vigato, S. Tamburini, and D. E. Fenton, Coord. Chem. Rev., 106, 25 (1990), and references cited therein.

2) M. Tanaka, M. Kitaoka, H. Ōkawa, and S. Kida, Bull. Chem. Soc. Jpn., 49, 2469 (1976); W. Kanda, M. Nakamura, H. Ōkawa, and S. Kida, Bull. Chem. Soc. Jpn., 55, 471 (1982), and references cited therein.

3) J. D. Crane, D. E. Fenton, J.-M. Latour, and A. J. Smith, J. Chem. Soc., Dalton Trans., 1991, 2979.
4) W. Kanda, W. Moneta, M. Bardet, E. Bernard, N. Debaecker, J. Laugier, A. Bousseksou, S. Chardon-Noblat, and J. -M. Latour, Angew. Chem., Int. Ed. Engl., 34, 588 (1995).

5) K. Kayatani, Y. Hayashi, M. Suzuki, and A. Uehara, Bull. Chem. Soc. Jpn., 67, 2980 (1994).

6) P. Kamaras, M. C. Cajulis, M. Rapta, G. A. Brewer, and G. B. Jameson, J. Am. Chem. Soc., 116, 10344 (1994).

7) J. H. Satcher, Jr., M. W. Droege, T. J. R. Weakley, and R. T. Taylor, Inorg. Chem., 34, 3317 (1995).

8) See for examples: J. B. Vincent, G. L. Olivier-Lilley, and B. A. Averill, Chem. Rev., 90, 1447 (1990), and references cited therein; R. E. Stenkamp, Chem. Rev., 94, 715 (1994), and references cited therein; A. L. Feig and S. J. Lippard, Chem. Rev., 94, 759 (1994), and references cited therein.

9) See for examples: K. A. Magnus, H. Ton-That, and J. E. Carpenter, Chem. Rev., 94, 727 (1994), and references cited therein.

10) a) V. McKee, J. V. Dagdigian, R. Bau, and C. A. Reed, J. Am. Chem. Soc., 103, 7000 (1981); b) V. McKee, M. Zvagulis, J. V. Dagdigian, M. G. Patch, and C. A. Reed, J. Am. Chem. Soc., 106, 4765 (1984).

11) a) Y. Nishida, M. Takeuchi, K. Takahashi, and S. Kida, Chem. Lett., 1983, 1815; b) Y. Nishida and S. Kida, J. Chem. Soc., Dalton Trans., 1986, 2633.

12) W. Mazurek, B. J. Kennedy, K. S. Murray, M. J. O'Connor, J. R. Rodgers, M. R. Snow, A. G. Wedd, and P. R. Zwack, Inorg. Chem., 24, 3258 (1985).

13) S. Wang, S. J. Trepanier, J. C. Zheng, Z. Pang, and M. J. Wagner, Inorg. Chem., 31, 2118 (1992).

14) M. Suzuki, H. Senda, M. Suenaga, T. Sugisawa, and A. Uehara, Chem. Lett., 1990, 923; M. K. Chan and W. H. Armstrong, J. Am. Chem. Soc., 113, 5055 (1991).

15) M. Suzuki, T. Sugisawa, and A. Uehara, Bull. Chem. Soc. Jpn., 63, 1115 (1990).

16) Y. Nishida, M. Takeuchi, H. Shimo, and S. Kida, Inorg. Chim. Acta, 96, 115 (1984); D. L. Jameson, C. L. Xie, D. N. Hendrickson, J. A. Potenza, and H. J. Schugar, J. Am. Chem. Soc., 109, 740 (1987); Q. Chen, J. B. Lynch, P. G. Romero, A. B. Hussein, G. B. Jameson, C. J. O'Connor, and L. Que, Jr., Inorg. Chem., 27, 2673 (1988); S. Menage, B. A. Brennan, C. J. Garcia, E. Münck, and L. Que, Jr., J. Am. Chem. Soc., 112, 6423 (1990); Y. Hayashi, M. Suzuki, A. Uehara, Y. Mizutani, and T. Kitagawa, Chem. Lett., 1992, 91.

17) Y. Hayashi, T. Kayatani, H. Sugimoto, M. Suzuki, K. Inomata, A. Uehara, Y. Mizutani, T. Kitagawa, and Y. Maeda, $J$. Am. Chem. Soc., 117, 11220 (1995).

18) F. E. Mabbs and D. J. Marchin, "Magnetisms and Transition Metal Complexes," Chapman and Hall, London (1975), p. 5.

19) G. M. Sheldrick, "SHELXS-86. A Program for Crystal Structure Determination," University of Göttingen, FRG (1986).

20) G. M. Sheldrick, "SHELX-76. A Program for Crystal Structure Determination," Cambridge University, Cambridge, UK (1976).

21) "International Tables for X-Ray Crystallography," Kynoch Press, Birmingham, England (1974), Vol. IV.

22) They are deposited as Document No. 69009 at the Office of the Editor of Bull. Chem. Soc. Jpn.

23) G. B. Deacon and R. J. Phillips, Coord. Chem. Rev., 33, 227 (1980).

24) T. R. Felthouse, E. J. Laskowski, and D. N. Hendrickson, Inorg. Chem., 16, 1077 (1977); T. R. Felthouse and D. N. Hendrickson, Inorg. Chem., 17, 1978 (1978).

25) P. J. Hay, J. C. Thibeault, and R. Hoffmann, J. Am. Chem. 
Soc., 97, 4884 (1975).

26) J. Comarmond, P. Plumere, J. -M. Lehn, Y. Agnus, R. Louis, R. Weiss, O. Kahn, and I. Morgenstern-Badarau, J. Am. Chem. Soc., 104, 6330 (1982), and references sited therein.

27) R. Hoffmann, J. Chem. Phys., 39, 1397 (1963).

28) R. H. Summerville and R. Hoffmann, J. Am. Chem. Soc.,
98, 7240 (1976).

29) The atomic parameters are as follows: $H_{i j}, \mathrm{Cu} 4 \mathrm{~s},-11.4$ $\mathrm{eV} ; \mathrm{Cu} 4 \mathrm{p},-6.06 \mathrm{eV} ; \mathrm{Cu} 3 \mathrm{~d},-14.0 \mathrm{eV}$; orbital exponents $\mathrm{Cu} 4 \mathrm{~s}$, 2.20; Cu 4p, 2.20; Cu 3d, 5.95 (0.5933)+2.30 (0.5744). The C, H, $\mathrm{N}$, and $\mathrm{O}$ parameters are the standard ones.

30) H. Oshio and U. Nagashima, Inorg. Chem., 31, 3295 (1992). 